\title{
OPEN Multisubstituted pyrimidines effectively inhibit bacterial growth and biofilm formation of Staphylococcus aureus
}

\author{
Riccardo Provenzani ${ }^{1,3}$, Paola San-Martin-Galindo ${ }^{2,3}{ }^{\text {, Ghada Hassan }}{ }^{1,3}$, Ashenafi Legehar ${ }^{1}$, \\ Aleksi Kallio $^{1}$, Henri Xhaard ${ }^{1}$, Adyary Fallarero ${ }^{2} \&$ Jari Yli-Kauhaluoma ${ }^{1 凶}$
}

Biofilms are multicellular communities of microorganisms that generally attach to surfaces in a self-produced matrix. Unlike planktonic cells, biofilms can withstand conventional antibiotics, causing significant challenges in the healthcare system. Currently, new chemical entities are urgently needed to develop novel anti-biofilm agents. In this study, we designed and synthesized a set of 2,4,5,6-tetrasubstituted pyrimidines and assessed their antibacterial activity against planktonic cells and biofilms formed by Staphylococcus aureus. Compounds $9 \mathrm{e}, 10 \mathrm{~d}$, and $10 \mathrm{e}$ displayed potent activity for inhibiting the onset of biofilm formation as well as for killing pre-formed biofilms of $S$. aureus ATCC 25923 and Newman strains, with half-maximal inhibitory concentration $\left({ }^{2} C_{50}\right)$ values ranging from 11.6 to $62.0 \mu \mathrm{M}$. These pyrimidines, at $100 \mu \mathrm{M}$, not only decreased the number of viable bacteria within the pre-formed biofilm by $2-3 \log _{10}$ but also reduced the amount of total biomass by $30-50 \%$. Furthermore, these compounds were effective against planktonic cells with minimum inhibitory concentration (MIC) values lower than $60 \mu \mathrm{M}$ for both staphylococcal strains. Compound 10d inhibited the growth of $S$. aureus ATCC 25923 in a concentration-dependent manner and displayed a bactericidal anti-staphylococcal activity. Taken together, our study highlights the value of multisubstituted pyrimidines to develop novel anti-biofilm agents.

Antimicrobial resistance is a recognized growing global threat that can affect the life of anyone at any age and currently it accounts for 700,000 deaths every year ${ }^{1}$. The number is estimated to rise to 10 million by 2050 and will be accompanied by an economic loss of about 100 trillion USD during the same period. Antibiotics are vital in treating bacterial infections, in preventing post-surgery infections, and they are also used in combination with cancer treatments ${ }^{2}$.

Bacteria can adhere to surfaces in a self-produced extracellular matrix to form multicellular structured communities known as biofilms. This allows them to tolerate chemical and environmental stresses ${ }^{3}$. Compared to the planktonic lifestyle, the biofilm state is challenging to counteract since it can withstand 10-1000 times higher doses of conventional antibiotics. Approximately $80 \%$ of nosocomial infections are biofilm-related and many of them are caused by the Gram-positive bacterium $S$. aureus ${ }^{4,5}$. This bacterium also causes respiratory tract and skin-related infections as well as food contamination ${ }^{6}$. Currently, there are no approved antimicrobial drugs specifically targeting biofilm infections ${ }^{7}$. Therefore, there is an urgent need for drugs that can either interfere with the biofilm formation at early stages or disrupt already established biofilms.

Since the 1980s, antibiotic development has strongly declined due to low economic incentives, low profit potential, and challenging regulatory requirements ${ }^{2,8}$. Meanwhile, current antibiotics are losing their efficacy due to the rising of antibiotic resistance to a dangerously high level. Therefore, new antibiotics that can antagonize multidrug-resistant bacteria are highly needed. During the past 20 years, pharmaceutical companies and academic research groups have mainly developed new antibiotic analogs by modifying the molecular scaffolds of existing drugs ${ }^{9}$. In fact, only four novel classes of antibiotics have entered the market during this period: oxazolidinones, ketolides, glycylcyclines and lipoglycopeptides ${ }^{9,10}$. With the current chemotherapeutic portfolio,

${ }^{1}$ Drug Research Program, Division of Pharmaceutical Chemistry and Technology, Faculty of Pharmacy, University of Helsinki, P.O. Box 56, (Viikinkaari 5 E), Fl-00014 Helsinki, Finland. '2Drug Research Program, Division of Pharmaceutical Biosciences, Faculty of Pharmacy, University of Helsinki, P.O. Box 56, (Viikinkaari 5 E), FI-00014 Helsinki, Finland. ${ }^{3}$ These authors contributed equally: Riccardo Provenzani, Paola San-Martin-Galindo, and Ghada Hassan. ${ }^{\square}$ email: jari.yli-kauhaluoma@helsinki.fi 


\section{Set I}

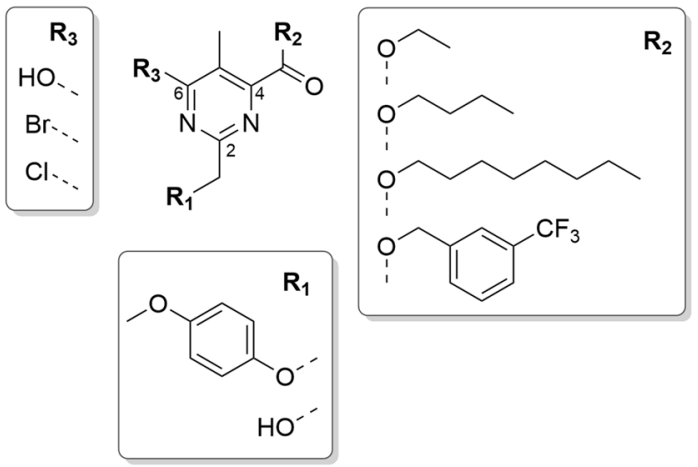

Set II

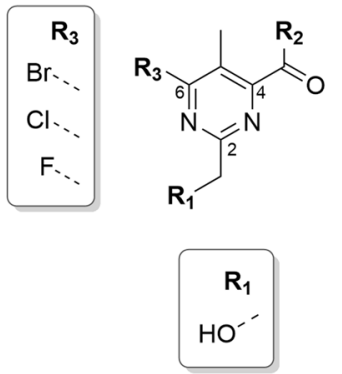

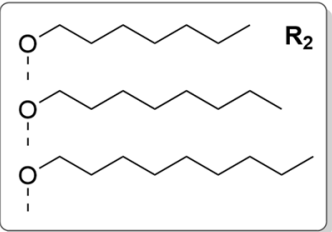

(1)

Figure 1. Sets I and II of the designed 2,4,5,6-tetrasubstituted pyrimidines with the scaffold and substituents.

the number of chemical modifications applicable to existing antibiotics are limited and they may prolong the efficacy of the known classes for up to a decade ${ }^{11}$. Hence, there is a need for new classes of antibiotics acting with different mechanisms and/or via new target sites.

Nitrogen-containing heterocyclic compounds are of great significance as they abound in nature and synthetic/semisynthetic derivatives exhibit a broad spectrum of biological activities ${ }^{12}$. Pyrimidines are heterocyclic aromatic compounds that are present in many natural and synthetic products and constitute essential building blocks for DNA and RNA biosynthesis ${ }^{13}$. Pyrimidine derivatives have shown a wide spectrum of biological activities including both non-chemotherapeutic, such as cardiovascular ${ }^{14}$ and anti-inflammatory ${ }^{15}$, as well as chemotherapeutic, such as anticancer ${ }^{16}$, antiviral ${ }^{17}$, and antibacterial ${ }^{18,19}$. In our previous work, we prepared a set of multisubstituted pyrimidines designed to target the $\mathrm{C} 1$ domain of protein kinase $\mathrm{C}^{20}$. However, a preliminary screening of these derivatives highlighted a potential antibacterial activity. Here, we report the design and synthesis of novel tetrasubstituted pyrimidines and their in vitro antimicrobial activity against planktonic and biofilm cells of $S$. aureus.

\section{Results and discussion}

Antimicrobial screening, design, synthesis, and structure-activity relationship analysis. We initially assessed a library of nineteen multisubstituted pyrimidines for antimicrobial activity against planktonic cells as well as biofilms formed by S. aureus ATCC 25923 at the high concentration of $400 \mu \mathrm{M}$ (all structures and biological data available in Supplementary Table S1). Of this library, seventeen compounds were already available, and their syntheses were previously reported by $\mathrm{us}^{20}$, while compounds $9 \mathbf{a}$ and $\mathbf{1 0 d}$ were prepared following the synthesis described below. Compound 10d was the most potent as it successfully inhibited the planktonic cells and biofilm viability by $\geq 90 \%$.

Based on this primary screening, we designed a first set of tetrasubstituted pyrimidines bearing: (1) a free or para-methoxyphenyl (PMP)-protected hydroxymethyl moiety in position C2, (2) an ester functionality in position C4 bearing linear alkyl substituents of different length (i.e. 2, 4, or 8 carbons) or a 3-(trifluoromethyl) benzyl substituent in C4, and (3) a phenolic hydroxy group, chloride or bromide in position C6 (Fig. 1, Set I). After completing the biological evaluation, we then fine-tuned a smaller set of derivatives focusing on the presence of (1) only the free hydroxymethyl moiety in C2, (2) an 1-heptyl or 1-nonyl ester in C4, and (3) also a fluoride in position C6 (Fig. 1, Set II).

All synthesized compounds presented in this study are grouped in the synthesis routes of Fig. 2. As previously reported ${ }^{20}$, we reacted the commercially available diethyl oxalpropionate (1) and 2-(4-methoxyphenoxy)acetamidine hydrochloride (2) in the presence of triethylamine (TEA) in absolute ethanol to obtain the pyrimidine 3 containing the PMP-protected hydroxymethyl moiety in position C2 (Fig. 2). The PMP protection is suitable to withstand the subsequent acid-catalyzed transesterifications of $\mathbf{3}$ in presence of the selected alcohols such as 1-butanol, 1-heptanol, 1-octanol, 1-nonanol, or 3-(trifluoromethyl)benzyl alcohol to give the derivatives $4 \mathbf{b}-\mathbf{f}$. Except for the 3-(trifluoromethyl)benzyl alcohol, the other alcohols reacted also in position C6, yielding enough disubstituted derivatives $\mathbf{5 b}-\mathbf{e}$ to be isolated for both testing and subsequent transformations. To explore the possible roles of different halogens on $\mathrm{C} 6$, compounds $\mathbf{3}$ and $\mathbf{4 b} \mathbf{b} \mathbf{f}$ were reacted with phosphoryl bromide or phosphoryl chloride in $\mathrm{N}, \mathrm{N}$-dimethylformamide (DMF) to give the corresponding halogenated derivatives $6 \mathbf{a}-\mathbf{e}$ and $7 \mathbf{a}-\mathbf{f}$, respectively. The bromination of $\mathbf{4 f}$ was not successful in the described conditions (Fig. 2, III). The chloride group of $7 \mathbf{e}$ was substituted with a fluoride when stirred with $\mathrm{KF}$ and $n$-tetrabutylammonium bromide in sulfolane as reported by Floersheimer and coworkers ${ }^{21}$. To remove the PMP protecting group, the intermediates $\mathbf{6 a}-\mathbf{e}, \mathbf{7 a}-\mathbf{f}, \mathbf{8 d}, \mathbf{5 b}$, and $\mathbf{5 d}$ were treated with ceric ammonium nitrate (CAN) in $\mathrm{MeCN} / \mathrm{H}_{2} \mathrm{O}$ to yield the final compounds $9 \mathbf{a}-\mathbf{e}, \mathbf{1 0 a}-\mathbf{f}, \mathbf{1 1 d}, \mathbf{1 2 b}$, and $12 \mathrm{~d}$, respectively.

To screen the biological activity of the first set of 21 pyrimidines, we determined their activity at $400 \mu \mathrm{M}$ against planktonic cells and biofilm formed by S. aureus ATCC 25923, with azithromycin as the control antibiotic in all viability assays (Supplementary Table S2). Compounds $\mathbf{9 d}$ and $\mathbf{1 2 b}$ exhibited the best activity as they caused over $80 \%$ inhibition of the viability of planktonic cells and over $81 \%$ of the biofilm formation of S. aureus 


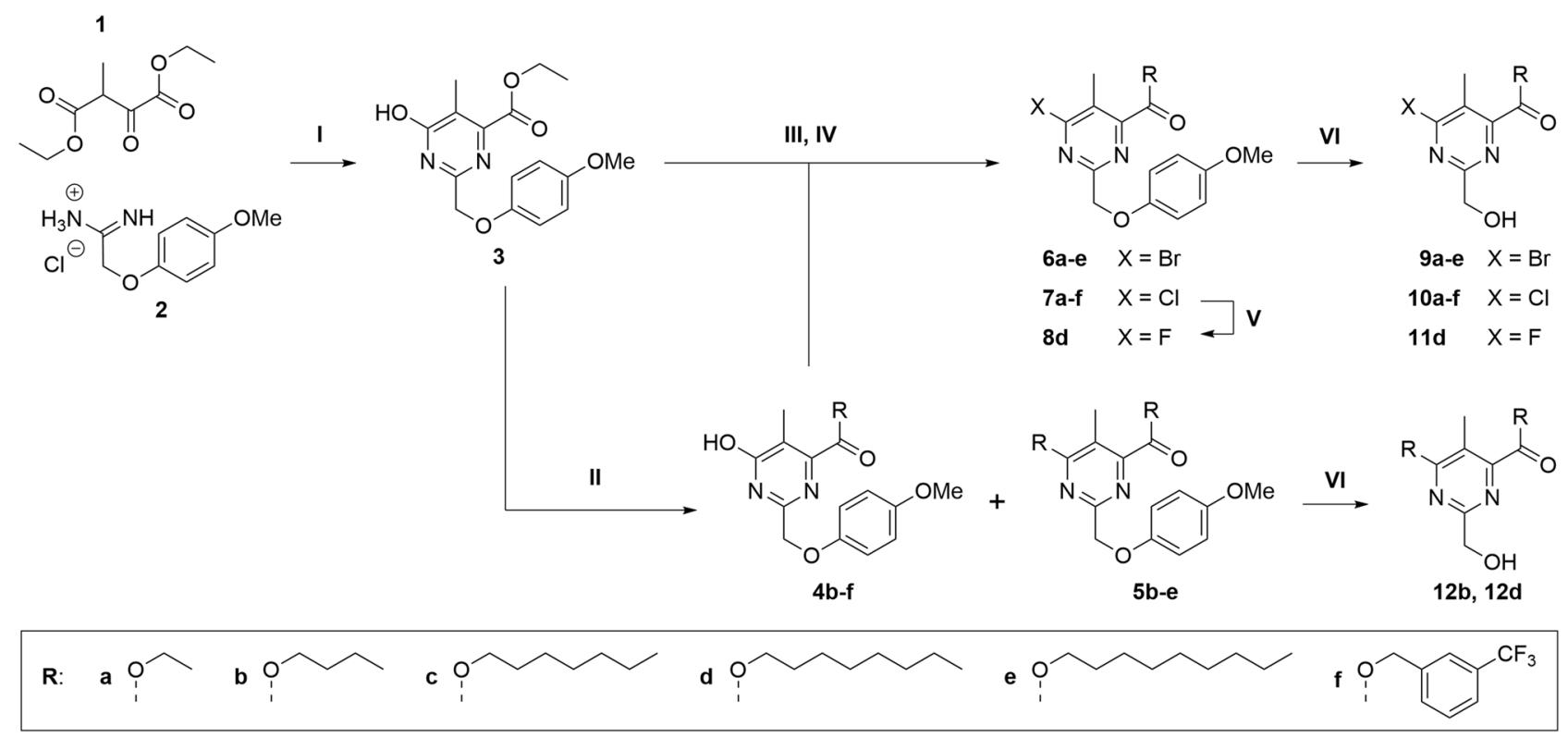

Figure 2. Synthesis and derivatization routes of sets I and II of 2,4,5,6-tetrasubstituted pyrimidines. Conditions: (I) TEA, EtOH, reflux, 2.5 h, 31\%; (II) alcohol, $\mathrm{H}_{2} \mathrm{SO}_{4}$ (cat.), $100{ }^{\circ} \mathrm{C}, 3-48 \mathrm{~h}, 18-58 \%$; (III) $\mathrm{POBr}_{3}, \mathrm{DMF}, \mathrm{MW}$ $90^{\circ} \mathrm{C}, 10-15$ min, 41\%-quant.; (IV) $\mathrm{POCl}_{3}$, DMF, MW $90^{\circ} \mathrm{C}, 10-15$ min, 72\%-quant.; (V) KF, TBAB, sulfolane, MW $150{ }^{\circ} \mathrm{C}, 2 \mathrm{~h}, 35 \%$; (VI) CAN, $\mathrm{MeCN} / \mathrm{H}_{2} \mathrm{O},-15^{\circ} \mathrm{C}, 10 \mathrm{~min}, 12-75 \%$.

\begin{tabular}{|c|c|c|c|c|c|c|}
\hline \multirow[b]{3}{*}{ Cmpd } & \multicolumn{4}{|c|}{ Staphylococcus aureus ATCC 25923} & \multirow{2}{*}{\multicolumn{2}{|c|}{\begin{tabular}{|l|}
$\begin{array}{l}\text { Staphylococcus aureus } \\
\text { Newman }\end{array}$ \\
Biofilm pre-exposure \\
\end{tabular}}} \\
\hline & \multicolumn{2}{|c|}{ Planktonic phase } & \multicolumn{2}{|c|}{ Biofilm pre-exposure } & & \\
\hline & Mean & $\pm \mathrm{SD}$ & Mean & $\pm \mathrm{SD}$ & Mean & $\pm \mathrm{SD}$ \\
\hline \multicolumn{7}{|c|}{ Inhibition percentage of bacterial viability (\%) } \\
\hline $9 \mathrm{c}$ & 1.42 & 12.44 & 8.58 & 5.32 & 49.32 & 7.48 \\
\hline $9 \mathrm{~d}$ & 58.22 & 15.34 & 51.77 & 3.62 & -3.12 & 3.40 \\
\hline $9 \mathrm{e}$ & 94.34 & 2.11 & 98.07 & 0.09 & 99.78 & 0.11 \\
\hline $10 \mathrm{c}$ & -3.35 & 2.31 & 6.32 & 3.32 & -10.56 & 7.00 \\
\hline $10 \mathrm{~d}$ & 92.51 & 4.98 & 97.85 & 2.25 & 97.85 & 3.00 \\
\hline $10 \mathrm{e}$ & 92.37 & 3.72 & 98.89 & 0.34 & 99.94 & 0.21 \\
\hline $11 \mathrm{~d}$ & 35.49 & 15.06 & 45.14 & 6.11 & -0.67 & 4.64 \\
\hline $12 b$ & 8.32 & 3.74 & 2.07 & 3.62 & 9.09 & 0.42 \\
\hline $\mathrm{Azm}^{*}$ & 99.58 & 0.09 & 96.97 & 0.44 & 99.26 & 0.05 \\
\hline
\end{tabular}

Table 1. Antimicrobial activity of the second set of 2,4,5,6-tetrasubstituted pyrimidines, including the most active compounds identified in the previous screenings against S. aureus ATCC 25923 and Newman clinical strains. All compounds were tested at $100 \mu \mathrm{M}$. Results are expressed as the mean value \pm standard deviation (SD) for three parallel replicates and two biological repetitions. ${ }^{*}$ Azithromycin (azm), the antibiotic used as the positive control, was tested at $400 \mu \mathrm{M}$.

ATCC 25923. The preliminary structure-activity relationship (SAR) analysis suggested that the PMP group did not contribute to the antimicrobial activity as all PMP-containing derivatives were inactive. Except for the C4-C6 dialkyl-substituted 12b, compounds bearing the ethyl, 1-butyl or 3-(trifluoromethyl)benzyl ester in C4 also lacked activity. The bromide-containing 9d exhibited slightly lower activity at preventing biofilm formation compared to the chloride-containing 10d. The halogen-related decrease in activity may be due to differences in steric properties or electron-withdrawing properties, which can affect the overall electron distribution in the conjugated ring system.

We explored further the SAR by synthesizing and testing a second set of derivatives comprising five additional compounds: 9c, 9e, 10c, 10d, and 11d (Table 1). As the most active 10d contains a 1-octyl ester, we introduced either 1-heptyl (10c, 9c) or 1-nonyl (10e, 9e) ester groups in position C4 to investigate the effect of \pm 1 carbon on the alkyl substituent. We also performed a chloride-fluoride replacement (11d) to investigate the effect of a smaller and more electronegative halogen in C6. In addition, to evaluate possible strain-specific activity, we tested the set II (9c, 9e, 10c, 10d, and 11d) and the most active compounds of set I (9d and 12b) at a lower concentration of $100 \mu \mathrm{M}$ against planktonic cells and biofilms formed by S. aureus ATCC 25923 and Newman (Table 1). 


\begin{tabular}{|c|c|c|c|c|c|c|}
\hline \multirow[b]{2}{*}{ Cmpd } & \multicolumn{2}{|c|}{ Planktonic bacteria } & \multirow[b]{2}{*}{$\mathrm{MBIC} \mu \mathrm{M}[\mathrm{mg} / \mathrm{L}]$} & \multicolumn{2}{|c|}{ Anti-biofilm activity $-\mathrm{IC}_{50}(\mu \mathrm{M})^{\mathrm{a}}$} & \multirow[b]{2}{*}{$\log R^{b} \log _{10}(C F U / m L)$} \\
\hline & $\mathrm{MIC} \mu \mathrm{M}[\mathrm{mg} / \mathrm{L}]$ & $\mathrm{MBC} \mu \mathrm{M}[\mathrm{mg} / \mathrm{L}]$ & & Pre-exposure & Post-exposure & \\
\hline \multicolumn{7}{|c|}{ Staphylococcus aureus ATCC 25923} \\
\hline $9 e$ & $40[14.9]$ & 80 [29.9] & $40[14.9]$ & $11.6(9.9-13.3)$ & $23.3(16.5-37.3)$ & 2.3 \\
\hline 10d & 60 [18.9] & $120[37.8]$ & 60 [18.9] & $23.6(19.6-28.9)$ & $54.9(50.8-59.1)$ & 2.8 \\
\hline $10 \mathrm{e}$ & 60 [19.7] & $120[39.5]$ & 60 [19.7] & $16.8(14.4-20.1)$ & $51.8(48.3-55.7)$ & 2.6 \\
\hline \multicolumn{7}{|c|}{ Staphylococcus aureus Newman } \\
\hline $9 \mathrm{e}$ & $40[14.9]$ & 60 [22.4] & 40 [14.9] & $29.4(27.8-30.5)$ & $31.9(26.0-38.0)$ & 1.8 \\
\hline 10d & 60 [18.9] & $90[28.3]$ & 60 [18.9] & $38.4(36.6-41.2)$ & $62.0(57.8-74.4)$ & 3.6 \\
\hline $10 \mathrm{e}$ & 60 [19.7] & $90[29.6]$ & $60[19.7]$ & $37.3(33.9-43.4)$ & $44.8(38.5-53.8)$ & 2.3 \\
\hline
\end{tabular}

Table 2. Antibacterial and anti-biofilm activity of the most active compounds against $S$. aureus ATCC 25923 and S. aureus Newman strains. ${ }^{\text {a The }} 95 \%$ confidence interval are shown in parentheses. ${ }^{\text {b Logarithm of }}$ reduction of biofilm burden on a pregrown biofilm. Compounds were tested at $100 \mu \mathrm{M}$.
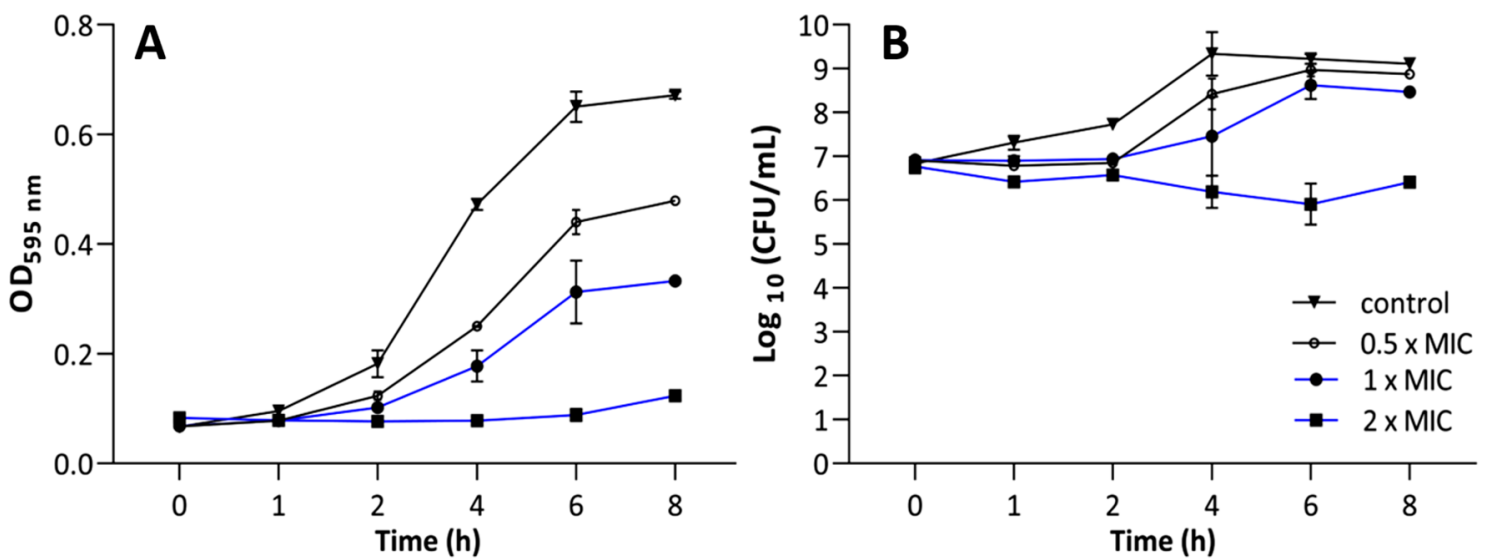

Figure 3. Time-kill assay of compound 10d for S. aureus ATCC 25923 during an 8-h incubation period represented by the bacterial growth curve (A) and number of CFUs (B). Data represent the mean of two biological repetitions with two technical replicates each \pm SEM.

The most active compounds, 9e, 10d, and 10e, inhibited the biofilm viability of both S. aureus ATCC 25923 and Newman strains by over $98 \%$. These three compounds also displayed antimicrobial activity against planktonic cells of S. aureus ATCC 25923 causing over $92 \%$ inhibition of viability at $100 \mu \mathrm{M}$. The fluoride in C6 did not improve the antimicrobial activity as $\mathbf{1 1 d}$ displayed a marginal inhibition compared to the chloride-containing 10d. The length of the alkyl ester in C4 deeply influenced the activity starting from eight carbons and shorter substituents caused loss of activity. Of note, compounds $\mathbf{9 e}$ and $\mathbf{1 0 e}$ displayed comparable results against biofilm and planktonic cells and it seems that the presence of the 1-nonyl ester chain contributed more to their activity independently of the nature of the halogen in C6. However, this was not the case for the 1-octyl ester-containing derivatives $9 \mathbf{d}$ and $\mathbf{1 0 d}$. At this concentration $(100 \mu \mathrm{M})$, the presence of the bromide in $9 \mathbf{d}$ reduced the overall activity but also highlighted a strain specificity compared to the chloride-containing 10d.

Antibacterial effect of 9e, 10d, and 10e on planktonic cells. To determine the antibacterial effects on suspended cells, we measured the minimal inhibitory concentration (MIC) and the minimal bactericidal concentration (MBC) values of the most active pyrimidines $\mathbf{9 e}, \mathbf{1 0 d}$, and $\mathbf{1 0 e}$ (Table 2). Interestingly, they exhibited equal MIC values in both staphylococcal strains and $9 \mathbf{e}$ prevailed as the most active antibacterial derivative with a MIC value of $40 \mu \mathrm{M}$. Comparing the MBC/MIC ratios, the three compounds displayed an equal ratio of 2 against $S$. aureus ATCC 25923, whereas a ratio of 1.5 against $S$. aureus Newman. This suggests that 9e, 10d, and 10e possess a bactericidal anti-staphylococcal behavior ${ }^{22}$.

To further investigate the bactericidal activity rate of these pyrimidines, we performed a time-killing assay using 10d against $S$. aureus ATCC 25923. We treated the cells with three concentrations $(0.5,1$, and 2 times the MIC value) within $8 \mathrm{~h}$. The time-kill curve shows a concentration-dependent bacterial death (Fig. 3A-B). No differences emerged between treated and non-treated cells during the first $2 \mathrm{~h}$, neither in optical density (OD) nor in number of colony-forming units (CFUs). After a 4-h exposure, 10d exhibited a concentration-dependent activity. The OD curves of $\mathbf{1 0 d}$ at $0.5 \times \mathrm{MIC}$ and $1 \times \mathrm{MIC}$ displayed lower OD values compared to the bacterial control; however, they still represent a bacterial growth pattern $(4-8 \mathrm{~h})$. The OD curve of $10 \mathrm{~d}$ at $2 \times \mathrm{MIC}$, instead, represents a maintained bacterial suspension during the tested time, which indicates an effective bacterial growth 
A

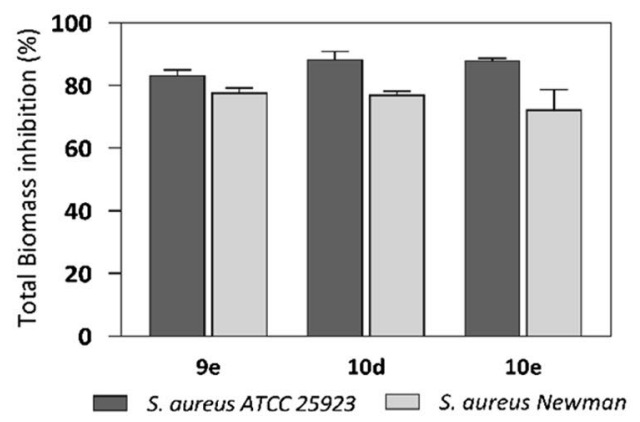

B After biofilm formation

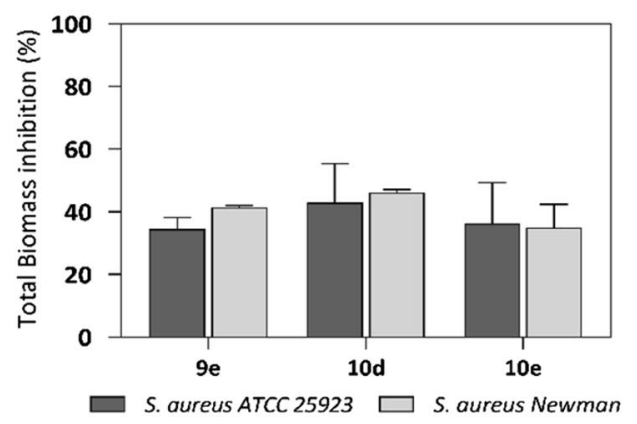

Figure 4. Anti-biofilm activities of $9 \mathrm{e}, \mathbf{1 0 d}$, and $10 \mathrm{e}$ at $100 \mu \mathrm{M}$ in the pre- (A) and post-exposure (B) assays. The total biomass was quantified using the crystal violet staining. Data represent the mean of two independent repetitions with three technical replicates each \pm SEM.

inhibition (Fig. 3A). Similarly, 10d at $2 \times$ MIC inhibited the bacterial growth of the initial inoculum as well as reduced the CFUs by over $2 \log _{10}$ from $4 \mathrm{~h}$ of exposure (Fig. 3B).

Anti-biofilm effect of $9 \mathrm{e}, \mathbf{1 0 d}$, and $10 \mathrm{e}$. We explored the activity of $9 \mathrm{e}, \mathbf{1 0 d}$, and $10 \mathrm{e}$ against $S$. aureus biofilms in both pre- and post-exposure inhibition assays by measuring the biofilm viability (Table 2). Of note, the minimal biofilm inhibitory concentration (MBIC) values of the pyrimidines equal their MIC values. For instance, at the MIC, 10d also inhibited by $90 \%$ the biofilm formation of both S. aureus strains in terms of viability (Table 2). Furthermore, the three pyrimidines exhibited MBIC values lower than their corresponding bactericidal (MBC) values.

Comparing the anti-biofilm potencies in terms of half maximal inhibitory concentration $\left(\mathrm{IC}_{50}\right)$, a twofold higher concentration of $\mathbf{9 e}$ and 10d reduced the viability of pre-formed S. aureus ATCC 25923 biofilms by $50 \%$, compared to the concentration needed to prevent the biofilm formation (Table 2). Biofilms can typically withstand 10-1000 times higher concentrations of conventional antibiotics compared to planktonic cells. Despite this, 9e, 10d, and 10e required only twice their MIC to effectively reduce by $>91 \%$ the viability of pre-formed biofilms. We measured inhibition potencies, in terms of biofilms viability, below $62 \mu \mathrm{M}$ for both tested staphylococcal strains. The bacterial growth states can dynamically switch between planktonic and biofilm stages depending on their nature and complex external driving forces ${ }^{23,24}$. Since our compounds act against both microbial states at relatively similar concentrations, they hold promise and could be further investigated as antimicrobial candidates.

To confirm the efficacy of these three compounds against pre-formed biofilms, we calculated the reduction of viable cells within the biofilm and measured the log reduction $(\log \mathrm{R})$ after a 24 -h exposure at $100 \mu \mathrm{M}$ (Table 2). Viable cell counts of the untreated control in the post-exposure assay were $2.4 \times 10^{8} \mathrm{CFU} / \mathrm{mL}$ and $2 \times 10^{8} \mathrm{CFU} /$ $\mathrm{mL}$ for S. aureus ATCC 25923 and Newman, respectively. Compounds 9e and 10e reduced close to and higher than $2-\log (>99 \%)$ the number of viable cell counts on pre-formed S. aureus biofilms. Compound 10d caused close to or more than a 3-log reduction in the viability of pre-formed S. aureus biofilms. Of note, a 3-log reduction corresponds to a killing efficacy of at least $99.9 \%$ of the viable biofilms. In addition, the viable cell density after treatment in the post-exposure phase suggested that the three compounds maintained a bacterial density of the planktonic suspension close to or more than $1 \times 10^{6} \mathrm{CFU} / \mathrm{mL}$ (Supplementary Fig. S1) in both staphylococcal strains. This demonstrates that these pyrimidines reduce the cell viability of established biofilms without considerably killing planktonic cells, and thus, this confirms their effect as potent anti-biofilm agents. Taken together, 10d emerged as the most potent anti-biofilm agent with the highest measured efficacy.

To investigate the effect of the new pyrimidines on Gram-negative biofilm-forming strains, we quantified the viable burden of two Pseudomonas aeruginosa strains (ATCC 9027 and ATCC 15442) after a 24-h exposure to 9e, 10d, and 10e at $400 \mu \mathrm{M}$ (Supplementary Fig. S2). None of the compounds showed significant inhibition in the number of viable cell count against either P. aeruginosa strain, thus we concluded that these tetrasubstituted pyrimidines preferably act against Gram-positive bacterial biofilms.

Effect of 9e, 10d, and 10e on the biofilm biomass. Once we confirmed the effectivity of these pyrimidines to reduce viable bacteria within the biofilm, we assessed the impact of $9 \mathbf{e}, \mathbf{1 0 d}$, and $10 \mathrm{e}$ at $100 \mu \mathrm{M}$ on the biofilm biomass by performing a crystal violet assay. The three pyrimidines inhibited the biofilm biomass of S. aureus ATCC 25923 and Newman by $80 \%$ and $70 \%$, respectively, compared to the untreated control, after an 18-h exposure (Fig. 4A). This suggests that the compounds prevent the biofilm formation not only in terms of viability (as shown in Table 1) but also in terms of total biomass. In addition, the compounds reduced the total biofilm biomass of established biofilm by $30-50 \%$, with $10 \mathrm{~d}$ showing the highest efficacy among them (Fig. 4B). Comparing the outcomes in pre-formed biofilms, the percentage of inhibition of the total biomass differed from the biofilm viability (which resulted in a $>90 \%$ reduction; Table 2 ). As the crystal violet stains both extracellular matrix and live/dead cells ${ }^{25}$, we next investigated specifically the proportion of live and dead cells in the biofilms.

By applying the live/dead fluorescence kit, SYTO 9/PI, we gained insights into the physiological state of biofilm cells upon exposure to the pyrimidines as it quantifies viable and dead bacteria differentiated by their 
A
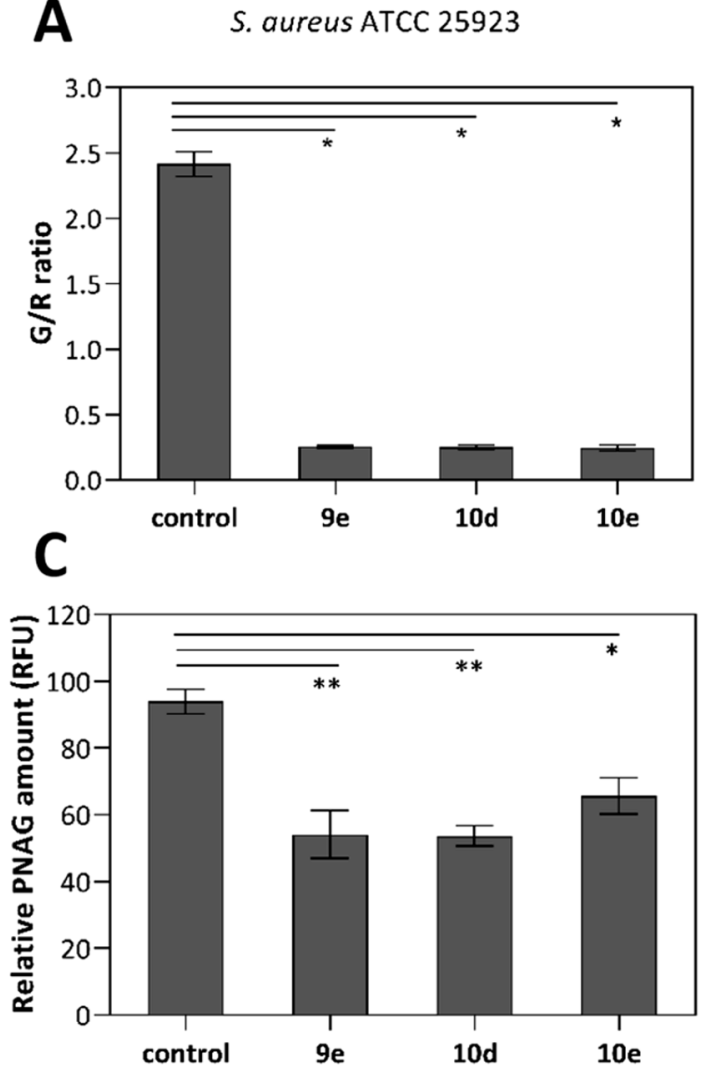

B

S. aureus Newman
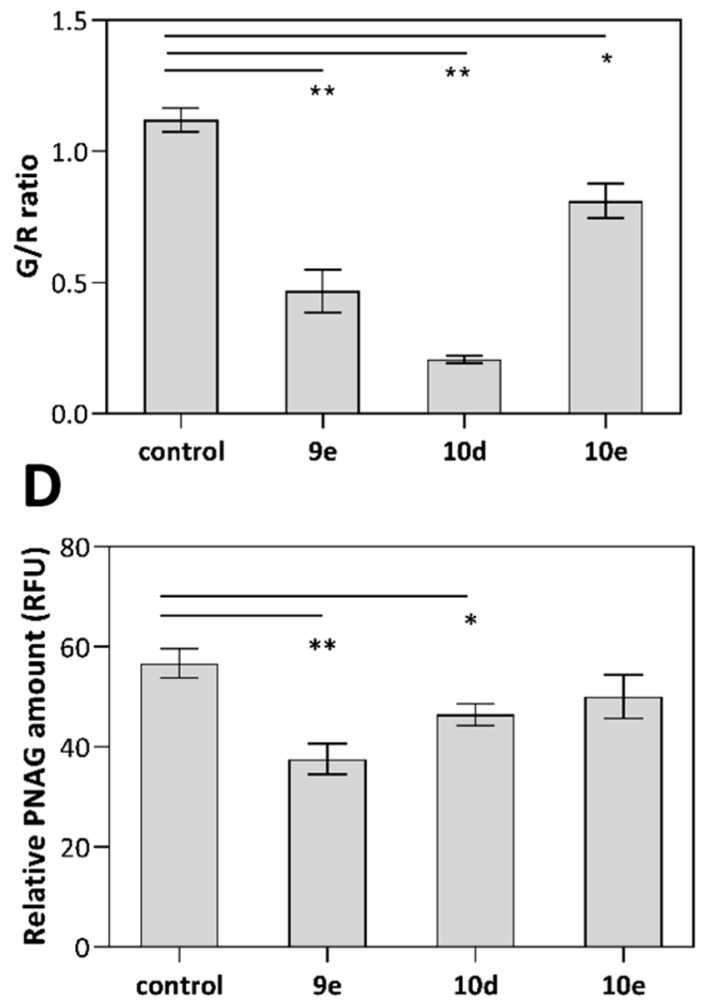

Figure 5. Effect of the pyrimidines $9 e, 10 d$, and 10e at $100 \mu \mathrm{M}$ after a 24 -h incubation with pre-formed biofilms of S. aureus ATCC 25923 (A and C) and S. aureus Newman (B and D). Quantification of the green-tored-fluorescence ratios (G/R), using SYTO 9 and PI fluorescent nucleic acid stains (A and B), and quantification of the poly- $N$-acetylglucosamine (PNAG) content (C and D) in terms of relative fluorescence units (RFU) measured by the wheat germ agglutinin Alexa Fluor 488 (WGA) staining. An unpaired $t$-test with Welch's correction was used to determine statistical differences $\left({ }^{*} p<0.05 ;{ }^{* *} p<0.01\right)$. Data represent the mean of two independent repetitions with at least two technical replicates each \pm SEM.

membrane integrity ${ }^{26}$. The fluorescent nucleic acid dyes, SYTO 9 and PI, penetrate bacterial cells differently. While the former labels all bacteria (green fluorescence), the latter labels only those with a damaged membrane (red fluorescence). We measured the green-to-red (G/R) fluorescence ratio after a 24-h exposure of pre-formed staphylococcal biofilms with $9 \mathrm{e}, \mathbf{1 0 d}$, and $10 \mathrm{e}$ at $100 \mu \mathrm{M}$. The two strains of untreated $S$. aureus biofilms displayed different G/R ratios. In untreated biofilms, S. aureus ATCC 25923 live cells predominated over dead cells (2.4 ratio; Fig. 5A) while the assay on $S$. aureus Newman yielded a more equal distribution of viable and non-viable bacteria (1.1 ratio; Fig. 5B). Our pyrimidines remarkably damaged bacterial cells of established biofilms as 9e, 10d, and 10e decreased the G/R ratio to roughly 0.25 and $0.21-0.81$ in S. aureus ATCC 25923 and Newman strains, respectively (Fig. 5A,B). Particularly, 10d showed the lowest G/R ratios ( 0.25 and 0.21$)$ in both staphylococcal strains and we visualized its efficacy to decrease viable biofilm cells of S. aureus ATCC 25923 by fluorescence imaging (Fig. 6). Altogether, these results support the anti-biofilm activity of the pyrimidines, at a low concentration of $100 \mu \mathrm{M}$, to disturb pre-formed biofilm.

To further characterize the activity of $9 \mathbf{e}, \mathbf{1 0 d}$, and $10 \mathrm{e}$ on the biomass of existing biofilms, we assessed their effect on the exopolysaccharide poly- $N$-acetylglucosamine (PNAG). PNAG, a positively charged polymer, is the major component of the extracellular polysaccharide substance (EPS) in S. aureus biofilms which is mediated by the expression of $i c a A D B C$-encoded enzymes ${ }^{27}$. Since the production of PNAG influences the cell-to-cell adhesion as well as the biofilm structure and integrity, PNAG is an attractive target to counteract biofilm-associated infections ${ }^{28}$. Here we quantified the bound PNAG residues in the EPS by using the wheat germ agglutinin-Alexa Fluor 488 fluorescent conjugate (WGA) ${ }^{29}$. In both $S$. aureus strains, $9 \mathbf{e}$ and $\mathbf{1 0 d}$ significantly inhibited the PNAG content of the EPS (Fig. 5C,D), and particularly, they reduced by nearly $50 \%$ the relative PNAG content of $S$. aureus ATCC 25923 biofilms in comparison to untreated biofilms. The PNAG content of $S$. aureus Newman biofilms, instead, were less susceptible to the pyrimidines as $\mathbf{9 e}$ and $\mathbf{1 0 d}$ decreased the WGA signal by about $40 \%$ and $23 \%$, respectively. Compound 10 e showed no significant inhibition. These results corroborate the effect of $9 \mathbf{e}$ and $10 d$ to disturb $S$. aureus biofilms as they act in existing biofilms not only by killing cells within the biofilms but also by inhibiting the biomatrix production (in terms of PNAG content), pointing to a significant fitness as anti-biofilm agents. 

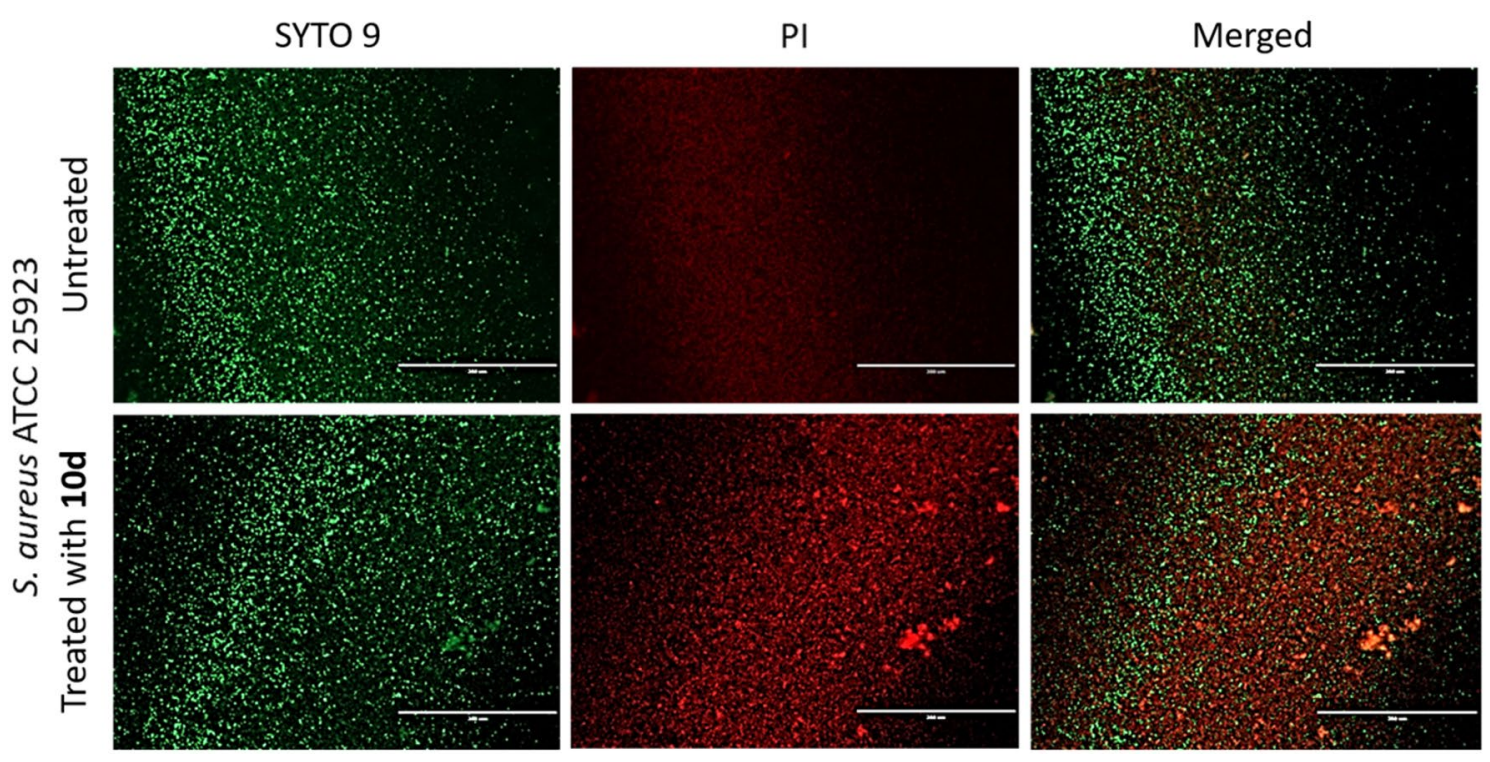

Figure 6. Fluorescence microscopy images of 18-h old S. aureus ATCC 25923 biofilms in a 96-well plate exposed to 10d at $100 \mu \mathrm{M}$ for $24 \mathrm{~h}$. Representative images of biofilms stained with LIVE/DEAD BacLight kit are displayed. Scale bars correspond to $200 \mu \mathrm{m}$.

Cytotoxicity of 9e, 10d, and 10e on human cells. We measured the in vitro cytotoxicity of these three most active pyrimidines against human Hep2 cell line, derived from epidermoid carcinoma cells, as an initial evaluation of unspecific toxicity. This is a common concern in the development of antimicrobials. Compounds 9e, 10d, and 10e exhibited cytotoxic activities when tested at the highest concentration of $100 \mu \mathrm{M}$ (see Supplementary Table S3). Compounds $9 \mathbf{e}$ and $\mathbf{1 0 e}$ maintained over $73 \%$ of cell viability when tested at $40 \mu \mathrm{M}$, which corresponds to the MIC of $9 \mathbf{e}$ and it is a concentration relatively higher than their determined potencies $\left(\mathrm{IC}_{50} \mathrm{~s}\right)$ to prevent the biofilm formation of $S$. aureus strains (Table 2). In contrast, 10d prompted a slightly higher unspecific cytotoxicity, under the same exposure conditions, as $55 \%$ of the cells remained viable.

Follow-up computational studies with $9 \mathrm{e}, \mathbf{1 0 d}$ and $10 \mathrm{e}$. To assess the novelty of compounds $9 \mathrm{e}, \mathbf{1 0 d}$, and 10e, we collected a dataset from ChEMBLv2 $7^{30,31}$ containing compound-bioactivity data for Gram-positive bacteria ( $n=21,922$ compounds; $n=2846$ assays; $n=56,670$ MIC data points). The histogram distribution of published $\log$ (MIC) showed that the activities of $\mathbf{9 e}, \mathbf{1 0 d}$, and 10e are average (Supplementary Fig. S3). We focused on identifying bioactive compounds sharing substructure or global similarity to our compounds. Neither close analogs (Tanimoto >0.7) nor pyrimidine-based compounds emerged, which supports the chemical novelty of $9 \mathrm{e}, 10 \mathrm{~d}$, and 10e. Among the distant active hits, several series contain an alkyl tail and an aromatic or nitrogen-containing ring, similar to our compounds. To note, the structure-activity relationships of compounds presented by Yarinich and coworkers hints to the optimal length of the alkyl tail containing at least seven carbons. This structural similarity extends our knowledge about antibacterial chemical frameworks and supports the findings presented in this manuscript. Considering the mode of action of some compounds extracted from the database, CHEMBL4166911 and CHEMBL3410371 (Supplementary Fig. S4) seem to exert their activity through interaction with the membrane ${ }^{32,33}$, and CHEMBL3417347, although it exhibits low intrinsic antibacterial activity, it seems to inhibit the efflux transporter NorA ${ }^{34}$. It appears that the mode of action of the molecular framework is not understood fully yet.

\section{Conclusion}

In this study, we designed and synthesized a novel set of 2,4,5,6-tetrasubstituted pyrimidines and highlighted the value of a suitable scaffold to develop antibacterial/anti-biofilm agents against Gram-positive staphylococcal strains. We identified three active compounds $(\mathbf{9 e}, 10 \mathrm{~d}$, and 10e), prepared via a four-step synthesis. These compounds inhibited the biofilm formation as well as disrupted the pre-formed biofilms of two strains of $S$. aureus (ATCC 25923 and Newman) in the low micromolar range. Furthermore, 10d effectively reduced the viability of pre-formed S. aureus Newman biofilm by $99.9 \%$ when tested at $100 \mu \mathrm{M}(31.48 \mathrm{mg} / \mathrm{L})$. We confirmed that these pyrimidines not only decreased the viability of cells within the $S$. aureus biofilms but also decreased the biofilm biomass, at least partially by reducing the PNAG content. We observed no significant reduction of viable cells of $P$. aeruginosa strains, thus suggesting that these compounds may act specifically against Gram-positive bacteria. Additionally, these pyrimidines displayed only marginal cytotoxicity on Hep2 cells at concentrations comparable to their corresponding pre-exposure $\mathrm{IC}_{50}$ values on $S$. aureus biofilm. Our findings enhance the promising role of multisubstituted pyrimidines in the research field of new anti-biofilm agents. 


\section{Methods}

Synthesis procedures. General information and experimental procedures of all synthesized compounds are available in Supplementary Information. All NMR spectra are available in Supplementary NMR Appendix.

Bacterial strains and growth conditions. Biofilm-forming clinical strains of S. aureus (ATCC 25923 and Newman) and P. aeruginosa (ATCC 15442 and ATCC 9027) were provided by the Faculty of Pharmacy, University of Helsinki, Finland. Bacterial strains were stored as cryogenic stocks $\left(-80^{\circ} \mathrm{C}\right)$ and were routinely propagated on either trypticase soy agar (TSA, Neogen) for S. aureus strains or Miller's Luria-Bertani agar (LBA, Fisher BioReagents) for P. aeruginosa strains. The bacterial cultures conditions are described in Supplementary Information.

Anti-biofilm and antibacterial assays. Biofilms were formed in flat-bottomed 96-well plate (Nunclon D Surface) by adding $200 \mu \mathrm{L}$ of $S$. aureus bacterial suspension (bacterial culture diluted to obtain $10^{6} \mathrm{CFU} / \mathrm{mL}$ ) per well, and plates were incubated under aerobic conditions for $18 \mathrm{~h}\left(37^{\circ} \mathrm{C}, 200 \mathrm{rpm}\right)^{35}$. The anti-biofilm activity of the compounds was primarily assessed against $S$. aureus strains (ATCC 25923 and Newman) under two modes of exposure (pre- and post-exposure), as previously described ${ }^{36,37}$. The compounds were initially tested at $400 \mu \mathrm{M}$ final concentration and further at $100 \mu \mathrm{M}$. TSB and DMSO $10 \%(\mathrm{v} / \mathrm{v})$ in TSB were included as the control solvents throughout the experiments, as earlier reported by $\mathrm{us}^{38}$. Azithromycin at $400 \mu \mathrm{M}$ was used as the viability control antibiotic. The bacterial viability of both planktonic phase and biofilms were quantified using the redox dye resazurin ${ }^{39}$. The procedures are described in Supplementary Information.

Determination of MIC and MBC. Bacteria were exposed to various dilutions of the selected compounds (0.01-400 $\mu \mathrm{M})$ using a 96-microtiter plate in similar conditions to the pre-exposure assay, as described above. To determine the MIC, after an 18-h incubation, the planktonic phase was transferred to a clean 96-microtiter plate $^{40}$. The MIC value was defined as the lowest concentration that prevented visible growth. To determine the $\mathrm{MBC}$, aliquots of $50 \mu \mathrm{L}$ from wells with non-visible bacterial growth were seeded onto TSA plates, which were incubated for $18-20 \mathrm{~h}$ at $37^{\circ} \mathrm{C}$. The $\mathrm{MBC}$ was defined as the lowest compound concentration that caused $\geq 3-\log$ reduction in the number of $\mathrm{CFU}$. Each test condition was performed using two biological repetitions, with each repetition undergoing three technical replicates.

Growth curve and time-killing studies. The dynamic of S. aureus ATCC 25923 treated with 10d was studied based on both the measurement of the OD at $595 \mathrm{~nm}$ as well as the quantification of viable cells at different time points $(0,1,2,4,6$, and $8 \mathrm{~h})$, when incubated at $37^{\circ} \mathrm{C}$ under shaking conditions. The procedure is described in Supplementary Information.

Determination of anti-biofilm potencies and MBIC. To determine the anti-biofilm potencies of 9e, 10d and 10e in both exposure modes of $S$. aureus ATCC 25923 and Newman strains, biofilms were exposed to the compounds using at least eleven concentrations serially diluted $(0.01-400 \mu \mathrm{M})$. These assays were performed using four replicates and two biological repetitions per tested compound. The cell viability was assessed using the resazurin dye, as mentioned above. The $\mathrm{IC}_{50}$ and $95 \%$ confidence interval were calculated by a nonlinear regression analysis (sigmoidal dose-response with variable slope) using GraphPad Prism 8.00 software. The MBIC was referred to the compound concentration that caused $\geq 90 \%$ inhibition of biofilm formation, compared to the untreated controls, when bacteria and compounds were added simultaneously ${ }^{41,42}$.

Antibacterial activity against gram-negative strains. The procedure is available in Supplementary Information.

Efficacy testing. The efficacy of the most active compounds was evaluated based on the logarithm reduction $\left(\log _{10} R\right)$ assay for both $S$. aureus strains, which was calculated by deducting the average $\log _{10}$ cell density $(\mathrm{CFU} / \mathrm{mL})$ in treated wells from the average $\log _{10}$ cell density in solvent control wells ${ }^{37,43}$. The 18 -h-old S. aureus biofilm was exposed to the compounds at a final concentration of $100 \mu \mathrm{M}$. The procedure for quantifying the number of viable colonies in the biofilm is detailed in Supplementary Information.

Biofilm biomass and biomatrix assays. The biofilm total biomass was quantified with crystal violet (Sigma-Aldrich), whereas the biomatrix was determined with the WGA probe (Alexa Fluor 488, Molecular Probes Inc.), as reported in our previous work ${ }^{29,35}$. The procedure is described in Supplementary Information.

Determination of live/dead ratio within pre-formed biofilms and fluorescence microscopy imaging. The 18-h-old biofilms were treated with the compounds (at $100 \mu \mathrm{M}$ ) in 96-microtiter plates, as described above. After a 24-h exposure, the planktonic suspension was removed, and biofilms were washed once with sterile PBS. The LIVE/DEAD Baclight Bacterial Viability kit (Molecular Probes Inc.) was used to determine the green-to-red fluorescence ratio as well as to visualize the live and dead cells by fluorescence microscopy ${ }^{44}$. The procedure is described in Supplementary Information.

Cytotoxicity study. The procedure is available in Supplementary Information. 
Statistical analysis. The assay performance was monitored by calculating the statistical parameters of screening window coefficient $Z^{\prime}$ factor, signal to noise $(\mathrm{S} / \mathrm{N})$, and signal to background (S/B) using Microsoft Excel 2016 software. Control wells containing bacteria and TSB were considered as maximum and minimal signal, respectively. For paired comparisons, an unpaired $t$-test with Welch's correction was applied $(p<0.05$ was considered statistically significant) and processed with GraphPad Prism 8.00 software.

\section{Data availability}

All data generated or analyzed during this study are included in this published article (and its Supplementary Information files).

Received: 2 October 2020; Accepted: 22 March 2021

Published online: 12 April 2021

\section{References}

1. O’Neill, J. Tackling Drug-Resistant Infections Globally: Final Report and Recommendations (2016). https://amr-review.org/sites/ default/files/160525_Final\%20paper_with\%20cover.pdf.

2. Ventola, C. L. The antibiotic resistance crisis: part 1: causes and threats. Pharm. Ther. 40, 277-283 (2015).

3. Rossolini, G. M., Arena, F., Pecile, P. \& Pollini, S. Update on the antibiotic resistance crisis. Curr. Opin. Pharmacol. 18, 56-60. https://doi.org/10.1016/j.coph.2014.09.006 (2014).

4. Bryers, J. D. Medical biofilms. Biotechnol. Bioeng. 100, 1-18. https://doi.org/10.1002/bit.21838 (2008).

5. Romling, U. \& Balsalobre, C. Biofilm infections, their resilience to therapy and innovative treatment strategies. J. Intern. Med. 272, 541-561. https://doi.org/10.1111/joim.12004 (2012).

6. Otto, M. Staphylococcal infections: mechanisms of biofilm maturation and detachment as critical determinants of pathogenicity. Annu. Rev. Med. 64, 175-188. https://doi.org/10.1146/annurev-med-042711-140023 (2013).

7. Sharahi, J. Y. et al. Advanced strategies for combating bacterial biofilms. J. Cell. Physiol. 234, 14689-14708. https://doi.org/10.1002/ jcp.28225 (2019).

8. Bartlett, J. G., Gilbert, D. N. \& Spellberg, B. Seven ways to preserve the miracle of antibiotics. Clin. Infect. Dis. 56, 1445-1450. https://doi.org/10.1093/cid/cit070 (2013).

9. Powers, J. H. Antimicrobial drug development-the past, the present, and the future. Clin. Microbiol. Infect. 10, 23-31. https://doi. org/10.1111/j.1465-0691.2004.1007.x (2004).

10. Timeline of antibiotics. https://en.wikipedia.org/wiki/Timeline_of_antibiotics. (last update, 21.12.2020).

11. Coates, A. R. M. \& Hu, Y. Novel approaches to developing new antibiotics for bacterial infections. Br. J. Pharmacol. 152, 1147-1154. https://doi.org/10.1038/sj.bjp.0707432 (2007).

12. Dua, R., Shrivastava, S., Sonwane, S. K. \& Srivastava, S. K. Pharmacological significance of synthetic heterocycles scaffold: a review. Adv. Biol. Res. 5, 120-144 (2011).

13. Sharma, V., Chitranshi, N. \& Agarwal, A. K. Significance and biological importance of pyrimidine in the microbial world. Int. J. Med. Chem. 2014, 202784. https://doi.org/10.1155/2014/202784 (2014).

14. Farghaly, A. M. et al. Design, synthesis, and antihypertensive activity of new pyrimidine derivatives endowing new pharmacophores. Med. Chem. Res. 28, 360-379. https://doi.org/10.1007/s00044-019-02289-6 (2019).

15. Ashour, H. M., Shaaban, O. G., Rizk, O. H. \& El-Ashmawy, I. M. Synthesis and biological evaluation of thieno [2',3':4,5] pyrimido $[1,2-b][1,2,4]$ triazines and thieno[2,3- $d][1,2,4]$ triazolo[ $[1,5-a]$ pyrimidines as anti-inflammatory and analgesic agents. Eur. J. Med. Chem. 62, 341-351. https://doi.org/10.1016/j.ejmech.2012.12.003 (2013).

16. Kumar, B. et al. Synthesis and biological evaluation of pyrimidine bridged combretastatin derivatives as potential anticancer agents and mechanistic studies. Bioorg. Chem. 78, 130-140. https://doi.org/10.1016/j.bioorg.2018.02.027 (2018).

17. Romeo, R. et al. Pyrimidine 2,4-diones in the design of new HIV RT inhibitors. Molecules 24, 1718. https://doi.org/10.3390/molec ules24091718 (2019).

18. Johar, M., Manning, T., Kunimoto, D. Y. \& Kumar, R. Synthesis and in vitro anti-mycobacterial activity of 5-substituted pyrimidine nucleosides. Bioorg. Med. Chem. 13, 6663-6671. https://doi.org/10.1016/j.bmc.2005.07.046 (2005).

19. Attia, M. I., Kansoh, A. L. \& El-Brollosy, N. R. Antimicrobial pyrimidinones II: synthesis and antimicrobial evaluation of certain novel 5,6-disubstituted 2-(substituted amino)alkylthiopyrimidin-4(3H)-ones. Monatsh. Chem. 145, 1825-1837. https://doi.org/ 10.1007/s00706-014-1253-2 (2014).

20. Provenzani, R. et al. Scaffold hopping from (5-hydroxymethyl) isophthalates to multisubstituted pyrimidines diminishes binding affinity to the C1 domain of protein kinase C. PLoS One 13, e0195668. https://doi.org/10.1371/journal.pone.0195668 (2018).

21. Floersheimer, A. et al. Diaryl urea derivatives useful for the treatment of protein kinase dependent diseases. US7652022B2 (2010).

22. Levison, M. E. Pharmacodynamics of antimicrobial drugs. Infect. Dis. Clin. North Am. 18, 451-465. https://doi.org/10.1016/j.idc. 2004.04.012 (2004).

23. Jefferson, K. K. What drives bacteria to produce a biofilm? FEMS Microbiol. Lett. 236, 163-173. https://doi.org/10.1016/j.femsle. 2004.06.005 (2004).

24. Flemming, H. C. et al. Biofilms: an emergent form of bacterial life. Nat. Rev. Microbiol. 14, 563-575. https://doi.org/10.1038/nrmic ro.2016.94 (2016).

25. Peeters, E., Nelis, H. J. \& Coenye, T. Comparison of multiple methods for quantification of microbial biofilms grown in microtiter plates. J. Microbiol. Methods 72, 157-165. https://doi.org/10.1016/j.mimet.2007.11.010 (2008).

26. Tawakoli, P. N., Al-Ahmad, A., Hoth-Hannig, W., Hannig, M. \& Hannig, C. Comparison of different live/dead stainings for detection and quantification of adherent microorganisms in the initial oral biofilm. Clin. Oral. Investig. 17, 841-850. https://doi.org/10. 1007/s00784-012-0792-3 (2013).

27. Arciola, C. R., Campoccia, D., Ravaioli, S. \& Montanaro, L. Polysaccharide intercellular adhesin in biofilm: structural and regulatory aspects. Front. Cell. Infect. Microbiol. 5, 7. https://doi.org/10.3389/fcimb.2015.00007 (2015).

28. Kropec, A. et al. Poly- $N$-acetylglucosamine production in Staphylococcus aureus is essential for virulence in murine models of systemic infection. Infect. Immun. 73, 6868-6876. https://doi.org/10.1128/Iai.73.10.6868-6876.2005 (2005).

29. Skogman, M. E., Vuorela, P. M. \& Fallarero, A. Combining biofilm matrix measurements with biomass and viability assays in susceptibility assessments of antimicrobials against Staphylococcus aureus biofilms. J. Antibiot. 65, 453-459. https://doi.org/10. 1038/ja.2012.49 (2012).

30. Gaulton, A. et al. ChEMBL: a large-scale bioactivity database for drug discovery. Nucleic Acids Res. 40, D1100-D1107. https://doi. org/10.1093/nar/gkr777 (2012).

31. Mendez, D. et al. ChEMBL: towards direct deposition of bioassay data. Nucleic Acids Res. 47, D930-D940. https://doi.org/10.1093/ nar/gky1075 (2019). 
32. Rahman, M. M., Shiu, W. K. P., Gibbons, S. \& Malkinson, J. P. Total synthesis of acylphloroglucinols and their antibacterial activities against clinical isolates of multi-drug resistant (MDR) and methicillin-resistant strains of Staphylococcus aureus. Eur. J. Med. Chem. 155, 255-262. https://doi.org/10.1016/j.ejmech.2018.05.038 (2018).

33. Yarinich, L. A. et al. Synthesis and structure-activity relationship of novel 1,4-diazabicyclo[2.2.2] octane derivatives as potent antimicrobial agents. Eur. J. Med. Chem. 95, 563-573. https://doi.org/10.1016/j.ejmech.2015.03.033 (2015).

34. Fontaine, F. et al. Boronic species as promising inhibitors of the Staphylococcus aureus NorA efflux pump: study of 6-substituted pyridine-3-boronic acid derivatives. Eur. J. Med. Chem. 95, 185-198. https://doi.org/10.1016/j.ejmech.2015.02.056 (2015).

35. Sandberg, M., Maattanen, A., Peltonen, J., Vuorela, P. M. \& Fallarero, A. Automating a 96-well microtitre plate model for Staphylococcus aureus biofilms: an approach to screening of natural antimicrobial compounds. Int. J. Antimicrob. Agents 32, 233-240. https://doi.org/10.1016/j.ijantimicag.2008.04.022 (2008).

36. Manner, S. et al. New derivatives of dehydroabietic acid target planktonic and biofilm bacteria in Staphylococcus aureus and effectively disrupt bacterial membrane integrity. Eur. J. Med. Chem. 102, 68-79. https://doi.org/10.1016/j.ejmech.2015.07.038 (2015).

37. Fallarero, A. et al. (+)-Dehydroabietic acid, an abietane-type diterpene, inhibits Staphylococcus aureus biofilms in vitro. Int. J. Mol. Sci. 14, 12054-12072. https://doi.org/10.3390/ijms140612054 (2013).

38. Oja, T. et al. Effective antibiofilm polyketides against Staphylococcus aureus from the pyranonaphthoquinone biosynthetic pathways of Streptomyces species. Antimicrob. Agents Chemother. 59, 6046-6052. https://doi.org/10.1128/Aac.00991-15 (2015).

39. Sandberg, M. E. et al. Pros and cons of using resazurin staining for quantification of viable Staphylococcus aureus biofilms in a screening assay. J. Microbiol. Methods 78, 104-106. https://doi.org/10.1016/j.mimet.2009.04.014 (2009).

40. Manner, S. \& Fallarero, A. Screening of natural product derivatives identifies two structurally related flavonoids as potent quorum sensing inhibitors against gram-negative bacteria. Int. J. Mol. Sci. 19, 1346. https://doi.org/10.3390/ijms19051346 (2018).

41. Gupta, A. Biofilm quantification and comparative analysis of MIC (minimum inhibitory concentration) \& MBIC (minimum biofilm inhibitory concentration) value for different antibiotics against E. coli. Int. J. Curr. Microbiol. Appl. Sci. 4, 198-224 (2015).

42. Manner, S., Goeres, D. M., Skogman, M., Vuorela, P. \& Fallarero, A. Prevention of Staphylococcus aureus biofilm formation by antibiotics in 96-microtiter well plates and drip flow reactors: critical factors influencing outcomes. Sci. Rep. 7, 43854. https://doi. org/10.1038/srep43854 (2017).

43. Pitts, B., Hamilton, M. A., Zelver, N. \& Stewart, P. S. A microtiter-plate screening method for biofilm disinfection and removal. J. Microbiol. Methods 54, 269-276. https://doi.org/10.1016/S0167-7012(03)00034-4 (2003).

44. Skogman, M. E., Vuorela, P. M. \& Fallarero, A. A platform of anti-biofilm assays suited to the exploration of natural compound libraries. JoVe https://doi.org/10.3791/54829 (2016).

\section{Acknowledgements}

The authors gratefully acknowledge financial support received from the Academy of Finland (Project No. 307464), the Strategic Research Council at the Academy of Finland (SRC-SUDDEN, Project No. 320210), the Jane and Aatos Erkko Foundation, and the Finnish Cultural Foundation. We thank Nina Sipari from Viikki Metabolomics Unit ViMU (Helsinki Institute of Life Science, University of Helsinki, Finland) for her expertise with the LC-MS analyses, as well as Eveliina Taavitsainen for the support in the performance of the cytotoxicity test. We also thank Dr. Andrew Neal for proofreading the manuscript.

\section{Author contributions}

R.P., P.S.-M.-G., and G.H. conceived and designed the study. R.P. and G.H. performed the design, carried out the syntheses, and performed the chemical characterization of the compounds. A.K. carried out part of the syntheses under the supervision of R.P. and G.H. P.S.-M.-G. performed the biological evaluation and characterization of the compounds. A.L. conducted the computational study. R.P., G.H., and P.S.-M.-G. prepared the manuscript with equal contribution. A.F., H.X, and J.Y.-K. supervised and revised the work. All authors reviewed and accepted the final version of the manuscript.

\section{Competing interests}

The authors declare no competing interests.

\section{Additional information}

Supplementary Information The online version contains supplementary material available at https://doi.org/ 10.1038/s41598-021-86852-5.

Correspondence and requests for materials should be addressed to J.Y.-K.

Reprints and permissions information is available at www.nature.com/reprints.

Publisher's note Springer Nature remains neutral with regard to jurisdictional claims in published maps and institutional affiliations.

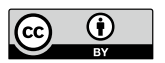

Open Access This article is licensed under a Creative Commons Attribution 4.0 International License, which permits use, sharing, adaptation, distribution and reproduction in any medium or format, as long as you give appropriate credit to the original author(s) and the source, provide a link to the Creative Commons licence, and indicate if changes were made. The images or other third party material in this article are included in the article's Creative Commons licence, unless indicated otherwise in a credit line to the material. If material is not included in the article's Creative Commons licence and your intended use is not permitted by statutory regulation or exceeds the permitted use, you will need to obtain permission directly from the copyright holder. To view a copy of this licence, visit http://creativecommons.org/licenses/by/4.0/.

(C) The Author(s) 2021 